\title{
An Energy Efficient and Low Cost Smart Recycling Bin
}

\author{
Geerish Suddul \\ Department of Business Informatics \& Software \\ Engineering \\ University of Technology, Mauritius \\ Republic of Mauritius
}

\author{
Nessen Nedoomaren Soobhen \\ Department of Business Informatics \& Software \\ Engineering \\ University of Technology, Mauritius \\ Republic of Mauritius
}

\begin{abstract}
This research work focuses on the application of IoT (Internet of Things) in the field of waste recycling. We propose an energy efficient and low cost prototype system for a recycling bin. In an attempt to ease sorting during waste disposal, the system identifies the type of waste material which is being discarded and automatically opens the corresponding bin. Being battery operated and providing real-time service imply that downtime due to power shortage must be kept at a minimum. Our approach focuses on a novel wake-up technique applied to an Arduino microcontroller which controls the opening and closing of the bin lids. Our technique reduces power consumption of the microcontroller by allowing it to be in deep sleep mode when not in use. This is achieved by concatenating a wake-up bit at the beginning of a request to wake up the sleeping microcontroller. It then, executes the request and goes back to sleep. Our experiments demonstrate an increase in battery life of more than 3 hours when compared to the standard full power mode usage. Also, we introduce QR (Quick Response) code to identify the type of waste products, which is less costly and more environmental friendly than RFID (Radio Frequency Identification) tag based solutions.
\end{abstract}

\section{Keywords}

Internet of Things (IoT), Energy Efficient System, Smart Systems, Waste Recycling, Deep Sleep Mode, Microcontroller, Sensor Networks

\section{INTRODUCTION}

All countries, irrespective of their surface area, geographical position and economic stability invest massively in waste management every year. Landfill which is the most common solution for waste disposal, is becoming scarce and needs to be replaced[1]. Among the different available solutions, recycling for paper, glass, metal and plastic promotes reuse and also reduces the risk of environmental damage. While there are different systems for collection of recyclable waste, we focus on the use of recycling bins. They are commonly available, and provide separate containers for different materials like paper, aluminum cans and plastic. While different strategies exist to demarcate each container, no verification can be done during disposal, which implies an expensive sorting process post collection.

Waste management has experienced a large number of innovative improvements over the years, but with the advent of smart cities, it has taken new dimensions. Research strive to build autonomous waste management systems, including smart bins, based on IoT technologies, using various sensors and microcontrollers. These systems rely mainly on batteries for the provisioning of power. Their adoption, therefore, implies minimum downtime due to battery maintenance, and various studies $[6,7,8]$ have expressed the need for a well balanced system in terms of amount of data collected and conservation of energy. More data being harvested means more precision but at the expense of energy. However if energy is conserved, less data is harvested leading to inexact information. Moreover, frequent battery replacement increases the running cost of the system, and has a higher negative impact of the environment by contributing to carbon dioxide emission and battery discharged waste.

Several smart waste recycling systems [3, 16-20] use RFID tags attached to products to effectively identify, as well as automate selection and sorting. Nevertheless, this approach contaminates the recycling process as RFID tags consist of antennas made of copper, aluminum or silver mixtures as well as plastic, glues and silicon circuit [3]. Moreover, while the tags are relatively inexpensive, their corresponding readers are costly [4]. Therefore, the introduction of RFID tags on consumer products on a large scale can contribute massively towards increasing electronic waste.

Driven by the above mentioned issues, our motivation is to identify a power efficient approach and a low cost, environmental friendly alternative to RFID tags in the context of smart waste recycling systems. We propose a prototype system for the automatic opening of recycling bin lids, based on the Arduino microcontroller using a QR code approach for identification.

\section{RELATED WORKS}

Sai et al.[5] attempt to reduce energy consumption by limiting data transfer between sensor nodes and a server connected to a wireless network. These nodes perform large amount of processing without any consideration for sleep mode. Therefore, high power is required and consumed during processing and we predict a short battery life time. The effect of node lifetime, energy consumption during listening mode, transmission, idle and sleep states are presented in [6], which concludes that data packet size is inversely proportional to the life time of the node. As data packet size increases, the lifetime of the battery decreases. Umbdenstock et al. [7] also mention that the most effective way to save power consists of diminishing the activity level of the node in favor of inactive or sleep states.

Folianto et al.[8] propose the use a low power radio to transmit the capacity level of a bin to a gateway which in turn sends the information to a back-end server. Their approach are based on a time interval of five minutes, after which the microcontroller and sensors are waken up to take new readings which are sent to the server. When no data is sensed, the sensors are put back to sleep mode. The main issue with this approach is the time interval. If it is too short, then power consumption increases, and if too long, the system can miss incoming requests. In an attempt to deal with this issue, 
Skocir et al. [9] use different timer values instead, and Kaur and Sood [10] calculates the timer interval base on the usage history and remaining battery energy level. Both approaches are still not appropriate when it comes to automatically opening the lid of a bin as its unrealistic to define a usage pattern.

Abedin et al.[11] recommend an energy efficient algorithm to schedule duty cycle for multiple sensors and appliances, based on three core stages: On-duty; Pre-off duty and Off-duty. The On-duty stage, allow devices to receive and transmit data at full capacity, and the Pre-off stage is activated when the device is idle for several minutes. It allows switching to Onduty to perform the required action or to Off-duty which sets it to either sleep or hibernate or power off. While on-duty mode consumes high level of energy, the pre-off/idle mode only provides a temporary reduction of power consumption before a wake-up call. Also, the system performs a lot of calculation at hardware level and as mention by [5], this approach consumes high energy and offloading of processing must be considered.

The work of Thakker and Narayanamoorthi [12] uses spectroscopy to separate biodegradable materials from nonbiodegradable waste. However the approach identifies only plastic through Near Infrared (NIR) reflectance spectroscopy. Their approach best works at factory level rather that at bin level which is the most convenient way as mentioned by Yann and Paul [4]. The diversity of waste materials complicates the recycling process, and proper classification is a necessity. Study [3] reveals that RFID is more appropriate for identification in smart recycling systems, as opposed to barcodes. This implies the usage of trillions of tags, which further complicates the recycling process.

Although different sleep mode patterns exist and are necessary for power saving, it is not apparent from our literature review that different modes have been applied or experimented. The most commonly used approach is the idle mode with time interval wake-up. We investigate the power saving modes in the next section.

\section{SLEEP MODES}

Sleep modes allow a microcontroller processor to pause program execution and close down internal components to reduce its current consumption. Sleep modes are therefore beneficial. When applied to the Arduino microcontroller, it shuts down some of its unused modules in the MCU according to Atmel datasheet [13]. The 8 bit AVR offers various sleep modes, which are listed from least to most power saving: SLEEP_MODE_IDLE (idle mode), SLEEP_MODE_ADC, SLEEP_MODE_PWR_SAVE, SLEEP_MODE_STAND_BY,

SLEEP_MODE_PWR_DOWN (power down mode).

We have devised a set of experiments to investigate power consumption of an Arduino Uno (ATmega328P processor) microcontroller during full mode (Not Optimized), idle mode and power down mode. A 9V Zinc-Carbon battery (6f22) with a capacity of $400 \mathrm{mAh}$ feeds power to the microcontroller and a multi-meter (DT9205A) is used to measure the current being consumed, without data communication. Figure1 depicts the current being consumed by each of the three modes.

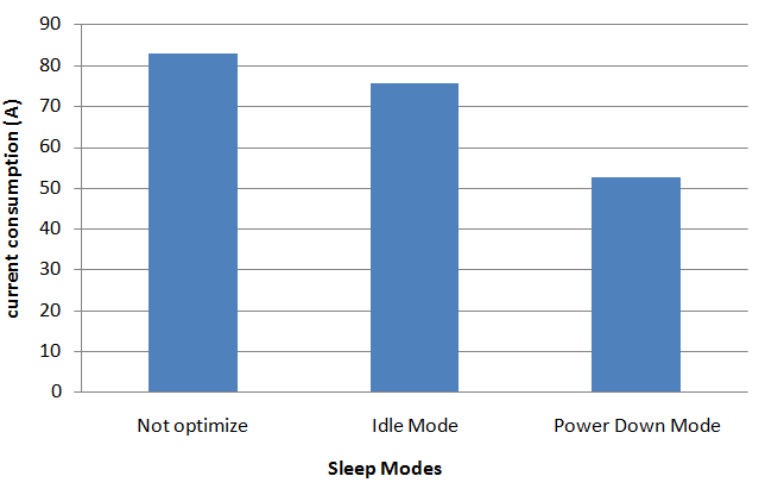

Figure 1: Current Consumption for different Sleep Modes

We observe that the idle mode has a current reduction of about $8 \%$ while the power down mode is around $36 \%$, when compared with the full power (Not Optimized) mode usage. In order to achieve this result, the power down mode disables many components, including serial mode communication. This is probably the main reason which explains its rare application, and therefore its unpopularity in our related works section. Serial communication is a very important aspect of IoT, and as such, any data sent to the Arduino microcontroller during power down mode is lost, including a wake-up call. The latter can be solved by one of the following interrupts: pin change, Watch Dog Timer (WDT) or Two Wired Interface (TWI). Waking up the micro controller through the pin change implies a button press to change the pin voltage from HIGH to LOW, which is not feasible in autonomous systems. The Watch Dog Timer uses an internal timer to cause a bit overflow which wakes up the Arduino after an interval. This time interval is controversial when it comes to power reduction. No results have been effectively demonstrated through the implementation of the TWI in an attempt to conserve energy.

\section{PROPOSED SOLUTION}

\subsection{System Architecture}

Figure 2 below depicts the main components of our prototype for a smart recycle bin system with automatic lid opening. The web camera captures the QR code on the waste product as an image and sends it for processing to the server, which consists of the $\mathrm{QR}$ reader middleware. It identifies the type of material from the received QR image. Following which the Arduino communication middleware, also a server component, sends the information to the microcontroller, which in turn activates the corresponding servo motor to open the appropriate bin lid. The power usage reduction is implemented at the microcontroller level by means of the SLEEP_MODE_PWR_DOWN, through the implementation our wake-up bit algorithm.

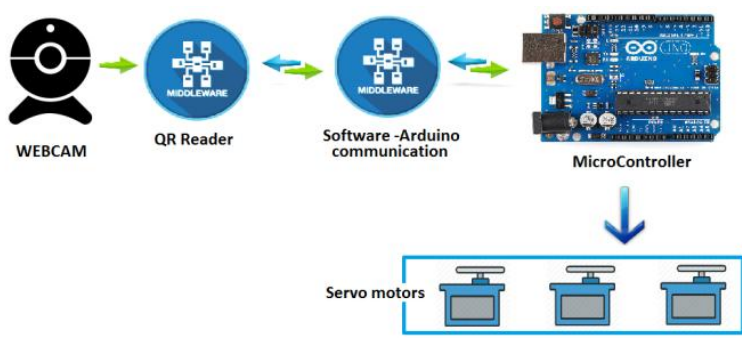

Figure 2: Proposed System Architecture 


\subsection{Low Power Model}

Initially the SLEEP_MODE_PWR_DOWN is invoked to put the microcontroller into deep sleep mode, which is recorded on the server. The microcontroller is activated by means of a wake-up bit, as opposed to a time interval. This wake-up bit is prepended to the original request at the server level before it is sent. Upon receiving the request, the wake-up bit interrupts the deep sleep mode of the microcontroller, which further reads the embedded command. The request is then executed in full power mode. While the microcontroller is in full power mode, the wake-up bit is skipped in all requests, as no wakeup call is necessary. Once a request is serviced, and no further requests are detected in the next 10 seconds, the server puts the microcontroller back in deep sleep mode. The activity diagram in Figure 3 depicts the flow from the server to the microcontroller. No specific overhead processing is implemented at the microcontroller level. It simply receives a command and sends a signal to open the corresponding lib by activating the corresponding servo motor. The server is responsible for decision making by means of the SoftwareArduino communication middleware depicted in Figure 2.

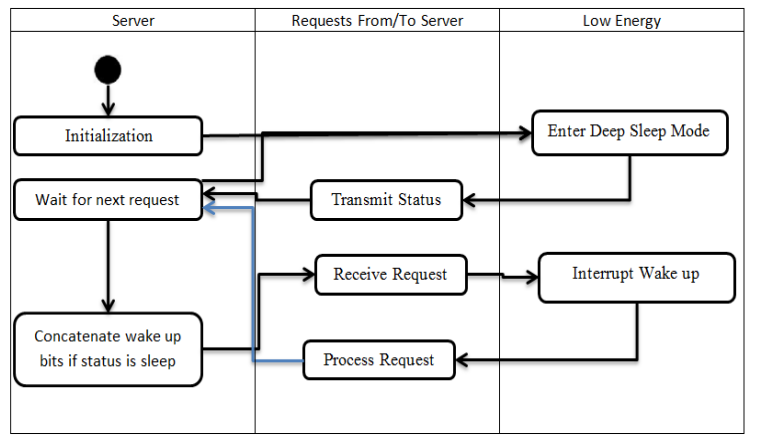

Figure 3: Activity Diagram

\subsection{Interrupt Mechanism}

Our interrupt mechanism is implemented by connecting the $\mathrm{Rx}$ (receiving) pin to the pin change interrupt. Initially both the Rx and interrupt pins are both set to $\mathrm{HIGH}(\mathrm{ON})$ and when the Arduino receives a request in form of data, the $\mathrm{Rx}$ pin drops from HIGH (ON) to LOW (OFF) and this change is reflected in the interrupt pin causing an interrupt signal to wake up the device. The first bit of data in the request sent to the microcontroller is our wake-up bit. However, once the system sleep mode is interrupted, the wake-up bit is lost, but the remaining bits corresponding to the original command is buffered for execution. Figure 4 depicts the interrupt mechanism by means of a sequence diagram.

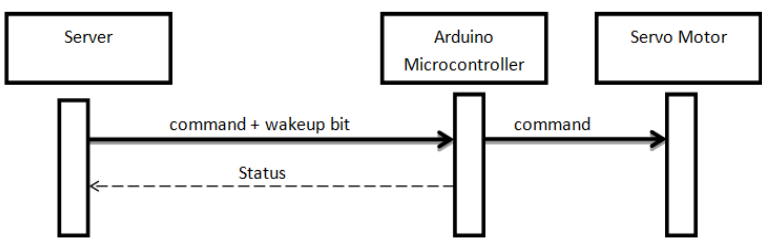

Figure 4: Interrupt Mechanism

\subsection{QR CODE Mechanism}

Instead of using RFID tags and its corresponding UHF reader, a web camera (webcam) and printed QR codes are introduced. The webcam is responsible for capturing the QR code which is printed on the waste product to identify its material composition. Webcams generally cost less than RFID readers and same applies for the production and printing of QR codes as opposed to RFID tags. Also, more information can be encoded in a QR code, as opposed to an RFID tag. In our prototype, we generate and print the $\mathrm{QR}$ code containing information pertaining to the type of material (paper, aluminum or glass). We assume the $\mathrm{QR}$ code is printed on the packaging of products. This QR code is then scanned by the webcam, which captures the image and sends it for processing to the $\mathrm{QR}$ reader component. The latter extract the hidden indentify code corresponding to the type of material. This code is sent to the Software-Arduino communication middleware to determine which servo motor to activate.

\section{EXPERIMENTS \& RESULTS}

Our experimental setup is based on our prototype system which is connected to the same equipments (microcontroller, battery and multi-meter) mentioned in section 3 . We firstly experiment with the prototype running at full power mode (Experiment 1), and secondly introduce our power efficient algorithm on the microcontroller (Experiment 2). The main differences being the microcontroller is initialized in deep sleep mode and the extra wake-up bit is sent to the microcontroller in experiment 2. Both experiments have been conducted over time intervals of 1 minute and the average current consumed, measured in milliampere $(\mathrm{mA})$ is shown in Table 1 below, together with the computed average consumption of the system, and the average time taken for each phase. The scenario is as follows: The system initializes and waits for an incoming request. At regular intervals of 20 seconds each, it receives a request from the server to activate the servo motor which opens for 4 seconds, and closes after. It then waits for the next request.

Table 1: Experimental Results

\begin{tabular}{|l|c|c|c|c|}
\hline Time Interval & \multicolumn{2}{|c|}{$\begin{array}{c}\text { Experiment 1: Full } \\
\text { Power Mode }\end{array}$} & \multicolumn{2}{c|}{$\begin{array}{c}\text { Experiment 2: } \\
\text { Proposed solution } \\
\text { (Power Down Mode) }\end{array}$} \\
\hline Seconds (s) & $\begin{array}{c}\text { Full } \\
\text { Mode }\end{array}$ & $\begin{array}{c}\text { Active } \\
\text { State + } \\
\text { Receiving } \\
\text { + servo } \\
\text { Execution }\end{array}$ & $\begin{array}{c}\text { Sleep } \\
\text { mode }\end{array}$ & $\begin{array}{c}\text { Active } \\
\text { State + } \\
\text { Receiving } \\
\text { + servo } \\
\text { Execution }\end{array}$ \\
\cline { 2 - 5 } & \multicolumn{2}{|c|}{$\begin{array}{c}\text { milliamps (mA) } \\
\text { milliamps (mA) }\end{array}$} \\
\hline 10 & 80.5 & & 41.4 & \\
\hline 20 & 78.5 & 84.5 & & 59.6 \\
\hline 30 & 77.0 & 82.0 & 41.3 & \\
\hline 40 & & 80.9 & 41.0 & 59.4 \\
\hline 50 & $\mathbf{7 8 . 7}$ & $\mathbf{8 2 . 5}$ & $\mathbf{4 1 . 2}$ & $\mathbf{5 9 . 3}$ \\
\hline 60 & $\mathbf{4 5}$ & $\mathbf{1 5}$ & $\mathbf{4 5}$ & $\mathbf{1 5}$ \\
\hline $\begin{array}{l}\text { Average } \\
\text { (mA) }\end{array}$ & & $\mathbf{7 7 . 4}$ & & $\mathbf{4 5 . 7}$ \\
\hline $\begin{array}{l}\text { Average } \\
\text { Time (s) }\end{array}$ & & & & \\
\hline $\begin{array}{l}\text { Average } \\
\text { Current } \\
\text { consumed by } \\
\text { system (mA) } \\
\text { in 60 s }\end{array}$ & & & & \\
\hline
\end{tabular}


It can be observed from the results in Table 1, that the Arduino microcontroller uses full power even though no request is received in experiment 1 . Whereas in experiment 2 , it goes to sleep mode when no request is sent, and waits for next request which wakes it up again. Using the $400 \mathrm{mAh}$ battery, we compute the battery lifetime to last around 5.17 hours in the first case, and up to 8.75 hours in the second case, as depicted in Figure 5. Therefore, our approach demonstrates saving of about $36 \%$ of power consumption and a significant gain of around $40.9 \%$ for the battery lifespan.

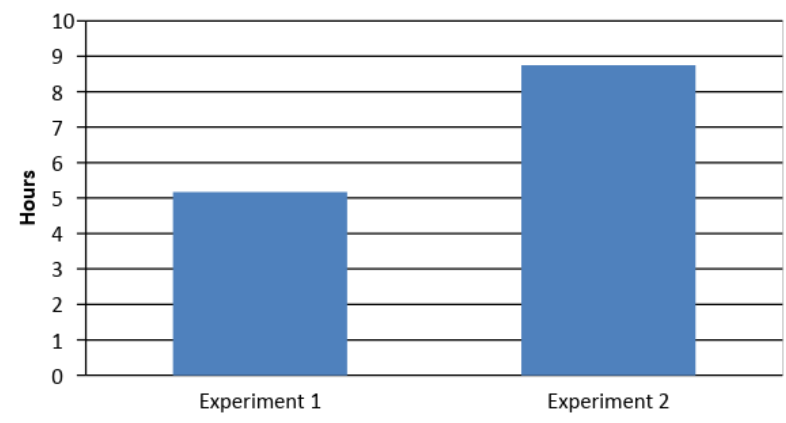

Figure 5: Battery Lifetime

Our second round of experiments consists of testing the accuracy and usability of the QR code approach, as a replacement for RFID tags. We have successfully generated the QR codes, and implemented the proposed system with three different servo motors corresponding to bin lids. At this stage, we have used an external source to power the webcam, and no measurement has been taken in comparison to power usage of UHF RFID readers yet.

\section{CONCLUSION}

Our research work presents an energy efficient technique, using power down mode on the Arduino microcontroller, applied to a recycling bin system. This is achieved with our automated wake-up bit technique, which follows an ondemand rather than time interval approach. It therefore eliminates unnecessary wake-up of the microcontroller, reducing its energy consumption. The battery life of the autonomous device is increased by nearly $40.9 \%$, which contributes to reduce maintenance downtime, and hence increases confidence level of the users as well as reduces electronic waste. We also introduce the $\mathrm{QR}$ code as an alternative to RFID tags, to identify the type of material and hence the system automatically opens the correct bin for disposal. Both approaches contributes towards a more environmental friendly waste disposal system.

The scope of the presented approach goes beyond its application to recycling systems. Our successful implementation of the deep sleep mode can be applied in various IoT and WSN real time systems to decrease maintenance downtime. This aspect contributes to increase system adoption by its users while reducing its running cost.

\section{FUTURE WORKS}

Our next step consists of investigating the power consumption of the web camera which is used for scanning the QR codes and compare it with the use of RFID readers. This will allow us to define the energy efficiency of the QR code components of in the prototype. In parallel, we are also devising an approach to complement or if necessary replace the webcam with a smartphone camera equipped with an application. The smartphone by means of its application and camera can scan the QR code and use low power Bluetooth to communicate directly with the microcontroller of the bin. We believe that modern smartphones have enough resources to host our proposed server components without compromising their features. At the same time the mobile application can provide users with appropriate details about the type of waste and sensitize towards reuse. Also, we are focusing on integrating more advanced features in the bins, by means of sensors, to automatically determine its capacity.

\section{REFERENCES}

[1] Catania, V. and Ventura, D. 2014 "An approach for monitoring and smart planning of urban solid waste management using smart-m3 platform" IEEE 15th Conference of Open Innovations Association FRUCT, pp. 24-31, 2014.

[2] Lundin, A. C. and Ozkil A. G. 2017 "Smart cities: A case study in waste monitoring and management", Proceedings of the 50th Hawaii International Conference on System Sciences, pp. 1392-1401.

[3] Thomas V. M., 2009 "A universal code for environmental management of products," Resources, Conservation and Recycling, vol. 53, no. 7, pp. 400-408, May 2009.

[4] Yann G. and Paul C. 2013 "A Smart Waste Management with Self-Describing objects", Leister, Wolfgang and Jeung, Hoyoung and Koskelainen, Petri. The Second International Conference on Smart Systems, Devices and Technologies (SMART'13).

[5] Sai, V. and Mickle, M. H. (2014) "Exploring energy efficient architectures in passive wireless nodes for IoT applications", IEEE Circuits and Systems Magazine, $14(2)$, pp. $48-54$

[6] Pawar M. S., Manore J. A. and Kuber M. M., 2011 "Life Time Prediction of Battery Operated Node for Energy Efficient WSN Applications", IJCST Vol. 2, Issue 4, Oct - Dec. 2011

[7] Umbdenstock E., Schafer F., Kleinsteuber F. and Meyer H., 2013, "Wake-Up-Receiver in energy efficient Wireless Sensor Networks for security applications" Agence National de la Recherche (ANR) Workshop Interdisciplinaire sur la Sécurité Globale, WISG2013 Université de Technologie de Troyes JAN 2013

[8] Folianto F., Low Y. S., and Yeow W.L., 2015 "Smartbin: Smart waste management system," IEEE, Tenth International Conference on Intelligent Sensors, Sensor Networks and Information Processing (ISSNIP), APR. 2015.

[9] Skocir, P., Zrncic, S., Katusic, D., Kusek, M. and Jezic, G., 2015 "Energy consumption model for devices in machine-to-machine system", 2015 IEEE 13th International Conference on Telecommunications (ConTEL).

[10] Kaur N., Sood S. K., 2015, "An Energy-Efficient Architecture for the Internet of Things", IEEE Systems Journal, no. 99, pp. 1-10, Oct. 2015.

[11] Abedin S. F., Alam M. G. R., Haw R., and Hong C. S., 2015, "A system model for energy efficient green-ioT network", International Conference on Information Networking (ICOIN), JAN. 2015. 
[12] Thakker, S. and Narayanamoorthi, R. (2015) 'Smart and wireless waste management' International conference on Innovations in Information, Embedded and Communication Systems ICIIECS, IEEE, pp. 1-4.

[13] Atmel, ATMEL 8-BIT MICROCONTROLLER WITH 4/8/16/32KBYTES IN-SYSTEM PROGRAMMABLE FLASH, "Power Management and Sleep Modes" p.62 [Online]http://ww1.microchip.com/downloads/en/Device Doc/Atmel-42735-8-bit-AVR-MicrocontrollerATmega328-328P_Datasheet.pdf Accessed: 2017

[14] Joshi J., Reddy P., Agarwal R., Bagga A., Bhargva A., 2016 "Cloud computing based smart garbage monitoring system", 3rd International comference on Electronic Design, ICED, Thailand, IEEE, pp. 70-75.

[15] Sharmin S.,Al-Amin S. T.,2016 "A Cloud-based dynamic waste management system for smart cities" 7th Annual Symposium on Computing for Development, ACM, pp. 20.
[16] Hannan M.A., Abdulla Al Mamun Md., Hussain A., Basri H., Begum., R.A., 2015, "A review on technologies and their usage in solid waste monitoring and management systems: Issues and challenges", Waste Management Journal, Volume 43, pp. 509-523.

[17] Hong, I., Park, S., Lee, B., Lee, J., Jeong, D. and Corporation, H.P. (2014) 'IoT-Based smart garbage system for efficient food waste management', The Scientific World Journal, 2014.

[18] Binder C. R., Quirici R., Domnitcheva S. and Stäubli B., 2008, "Smart Labels for Waste and Resource Management" Journal of Industrial Ecology, 12: 207 228

[19] Kwangho J. and Sabinne L., 2015 "A systematic review of RFID applications and diffusion: key areas and public policy issues", Journal of Open Innovation: Technology, Market, and Complexity (2015) 1:9 